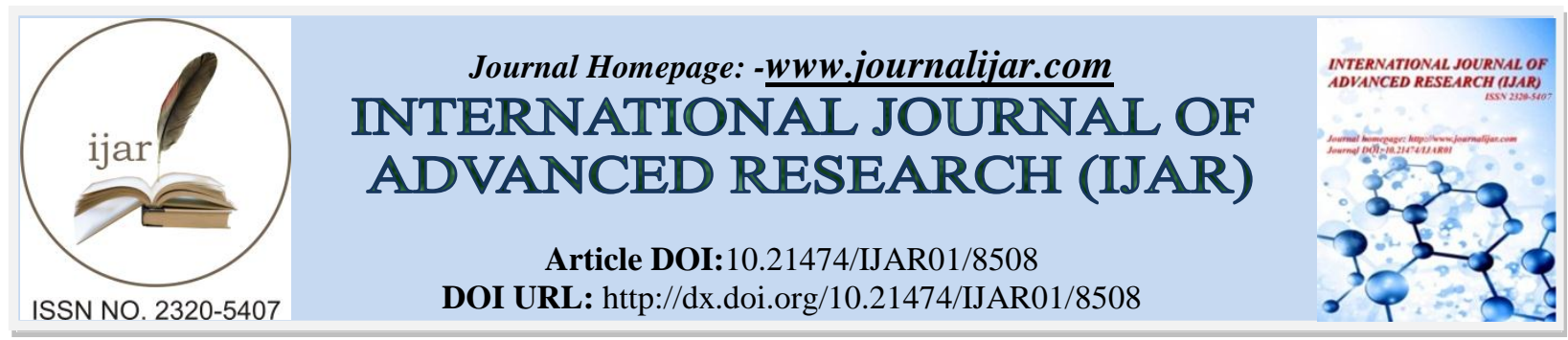

RESEARCH ARTICLE

\title{
ISOLATION AND IDENTIFICATION OF SOME RHIZOBIAL BACTERIA FROM FABA BEAN PLANTS UNDER EGYPTIAN SOIL CONDITIONS.
}

\section{Zeinat M. Kamel ${ }^{1}$, Mahmoud M. Nassef ${ }^{2}$ and Kholod I. Khalifa ${ }^{3}$.}

1. Microbiolody department, Faculty of science, Cairo university, Giza, Egypt.

2. Microbiolody department, Soil, Water and Environment Research Institute, Agricultural Research Center, Giza, Egypt.

3. Microbiolody department, Faculty of science, Cairo university, Giza, Egypt.

\section{Manuscript Info}

(..........................

Manuscript History

Received: 05 December 2018

Published: February 2019

Key words:-

Behaviour, determinant, factor, health, seeking.
Final Accepted: 07 January 2019

\section{Abstract}

Legumes are the most important source of human food and forage. Legumes are able to provide the benefits of nitrogen fixation thanks to a strain of soil bacteria.

Faba bean is the most important food legume. Moreover, it plays an important role in sustaining the productivity of the farming system through fixation of atmospheric nitrogen .

Sixteen rhizobial isolates were collected from the root nodules in various locations under different soil conditions.

Data indicated that most of the obtained isolates were superior compared to reference strains used .

All rhizobial isolates tested were fast growing on YEMA medium, noncapsulated, short rods, Gram negative and motile. Cell size ranged from 1 to $4 \mathrm{~mm}$.

According to various $\mathrm{NaCl}$ Concentrations (from 0.0 to 6.5\%), most of the tested isolates have a positive growth up to $4.5 \%$, but both isolates K-ISORH7 and K-ISORH15 gave good growth at $6.0 \% \mathrm{NaCl}$ Concentration.

The most potent isolates were K-ISORH7 and K-ISORH15 which recorded highest number of nodules $(172,188)$ for faba bean variety $\mathrm{V} 1$ and $(122,125)$ for V2, nodules dry weight $(597,607) \mathrm{mg}$ per plant for V1 and $(395,607) \mathrm{mg}$ per plant with V2 ,in the same order as compares to all tested isolates and strains. It took the same trend for the rest of parameters .

Genetically, according to 16S rRNA sequence, isolates were identified as Rhizobium sp. By 100\% similarity with HR7(KM276562) and strain SEMIA 439 (MH236581) for K-ISORH7 and K-ISORH15 respectively with accession numbers LC431741 and LC431742 in the same order.

Copy Right, IJAR, 2019,. All rights reserved.

\section{Introduction:-}

Recently in the $21^{\text {st }}$ century is marked by global scarcity of water resources, environmental pollution and elevated salinity levels of soil and water. Increasing human population and reduction in land available for cultivation are the 
main threats for agricultural sustainability. Many environmental stress factors like soil salinity, drought, extreme temperatures, high winds and flood have affected the production and cultivation of agricultural crops, among these; salinity of soil which is one of the most harmful environmental stresses, which causes wide losses in cultivated land area, crop productivity as well as its quality (Yamaguchi and Blumwald, 2005, Shahbaz and Ashraf, 2013).

Microorganisms would play a significant role in this respect. If we could take advantage of their unique properties like tolerance to saline conditions, bio-control potential, genetic diversity, production of plant growth promoting hormones, synthesis of compatible solutes and their interaction with crop plants (Pooja and Rajesh, (2015) .

Legumes are considered the second most important source of human food and animal forage after cereals. That is through nitrogen fixation that legumes provide plant tissue that is high in protein. In agricultural practice, legumes are used as an organic way to adjust the soil and reduce crop nutrient deficiency.

Legumes improve soil quality by increasing soil organic matter, improving soil porosity and structure, recycling nutrients, decreasing soil $\mathrm{pH}$, diversifying microorganisms and alleviating disease problems. Planting legumes as a cover crop also helps in reducing soil erosion.

Faba bean is considered the most significant food legume. It comprises a major part of the daily diet for the population. It also provides a major portion of the protein requirement. Moreover, it plays an important role in sustaining the productivity of the farming systems through the fixation of atmospheric nitrogen. Faba bean production is faced by many constrains like poor management practices, low yielding traditional cultivars, limited cultivable land and insect pest and diseases damage.

Developing countries use cultivated faba bean as human food in and as animal feed, mainly for pigs, horses, poultry and pigeons in industrialized countries. It can be used as a vegetable, green or dried, fresh or canned. It is a common breakfast food in the Middle East, Bond et al., (1985).

The present work aims to improve crop salt tolerance and enhance nitrogen fixation as well as growth of faba bean plants by isolation and identification of the most potent rhizobacteria under saline soil condition.

\section{Merials and methods:-}

\section{Collection and isolation of rhizobial isolates:-}

Rhizobia were isolated from root nodules according to the methods described by Somasegran and Hoben, (1994). Nodules were surface sterilized with $3 \% \mathrm{NaOCl}$ for 4 minutes then rinsed five times with sterile water and then crushed in a drop of sterile water on sterilized petri dish. A loop full of the crushed nodules was streaked on yeast extract mannitol agar (YEMA) medium containing congored pigment to ensure the purity of growth, and then the plates were incubated at 28o C. After 3-7 days individual colonies appearing over proved were re-streaked onto tubes containing YEMA medium and stored until the time of processing. Sixteen rhizobial isolates were collected from the root nodules of faba bean (Vicia faba L.) plants planted in 8 Egyptian governorates, table (1).

Table1:-Codes of different rhizobial isolates and their location in Egypt

\begin{tabular}{|l|l|l|}
\hline Number & Location & Code \\
\hline 1 & El-Menoufia & K-ISORH1 \\
\hline 2 & Qena & K-ISORH2 \\
\hline 3 & Qena & K-ISORH3 \\
\hline 4 & Matana & K-ISORH4 \\
\hline 5 & Matana & K-ISORH5 \\
\hline 6 & El-Menoufia & K-ISORH6 \\
\hline 7 & Giza & K-ISORH7 \\
\hline 8 & Kalubia & K-ISORH8 \\
\hline 9 & El-Minia & K-ISORH9 \\
\hline 10 & Nubaria, El-Behaira & K-ISORH10 \\
\hline 11 & El-Dakahlia & K-ISORH11 \\
\hline 12 & Kalubia & K-ISORH12 \\
\hline
\end{tabular}




\begin{tabular}{|l|l|l|}
\hline 13 & Kalubia & K-ISORH13 \\
\hline 14 & Giza & K-ISORH14 \\
\hline 15 & Baniswaif & K-ISORH15 \\
\hline 16 & Assiut & K-ISORH16 \\
\hline
\end{tabular}

\section{Laboratory experiments:-}

Morphological characteristics of rhizobial isolates:-

Pure colonies of rhizobial isolates were examined microscopically to determine cell shape and Gram reaction, whereas motility was tested in liquid culture. Morphology of colonies was determined - for each isolate which streaked on solid YEMA medium and incubated for 3 days at $28^{\circ} \mathrm{C}$ - by using binuclear microscope to evaluate colony size, transparency and viscosity, according to Somasegran and Hoben (1994).

\section{Salinity tolerance:-}

Gradient salt concentrations of $\mathrm{NaCl}(0.0,0.5,1.0,1.5,2.0,2.5,3.0,3.5,4.0,4.5,5.0,5.5,6.0$ and $6.5 \%)$ of $\mathrm{NaCl}$ were prepared in YEMA broth medium to evaluate the tolerance of obtained rhizobial isolates to salinity compared to reference Rhizobium strains namely ICARDA 441, ICARDA 481 and ARC 202, the three reference strains were kindly provided by Biofertilizers Production Unit, Agricultural Microbiology Department, Soil, Water and Environment Research (SWERI), Agriculture research center (ARC) Giza, Egypt, table (2).

Different concentrations of $\mathrm{NaCl}$ were inoculated by $1 \mathrm{ml}$ containing ca $10^{9} \mathrm{cfu}$ of new rhizobia cultures and incubated at $28^{\circ} \mathrm{C}$ for 168 hours and counting the appeared colonies on perti dishes containing YEMA medium.

Table 2:-Codes and source of the references strains

\begin{tabular}{|c|c|c|}
\hline Number & Location & Code \\
\hline 1 & SWERI & ARC-202 \\
\hline 2 & SWERI & ICARDA-441 \\
\hline 3 & SWERI & ICARDA-481 \\
\hline
\end{tabular}

Effect Salicylic acid on bacterial growth:-

The direct effect of SA at different concentrations on the growth of the most potent isolates was in vitro evaluated. Conical flasks each containing $150 \mathrm{ml}$ of sterilized YEMA medium were artificially seeded before solidifying with 5 $\mathrm{ml}$ of a $48 \mathrm{hr}$ old YEMA broth of tested bacteria. Concentrations at $(0.0,0.1,0.5,0.75,1.0,1.25,1.5,2.0,4.0,8.0$, 12.0, 16.0 and 20.0) $\mathrm{mM}$ of each SA were evaluated.

Five Petri dishes were used as replicates for each particular treatment as well as the control. All dishes were incubated at $28^{\circ} \mathrm{C}$ for $72 \mathrm{hr}$ then examined for bacterial growth.

\section{Media used:}

Yeast extract mannitol broth medium (Vincent, 1970):

It was used for the cultivation and maintenance of rhizobial isolates and strains. Its composition was as follows: mannitol (10.0 g/L), yeast extract (1.0 g/L), K2HPO4 (0.5 g/L), MgSO4.7H2O (0.2 g/L), NaCl (0.1 g/L), distilled water $(1000 \mathrm{ml})$ and the $\mathrm{pH}$ was adjusted to 7 .

\section{Screening for most potent isolates:-}

A pot experiment was carried out under given conditions of greenhouse to study the ability of tested 16 isolates to form nodules on faba bean compared with the three reference rhizobial strains (ICARDA 441, ICARDA 481 and ARC 202).

Two faba bean varieties [Giza 843 (V1) and Sakha 1 (V2)] were used. The two faba bean varieties were kindly obtained from department of legume crops, field crops research institute, ARC, Giza, Egypt.

Pure cultures of 16 rhizobial isolates and 3 reference strains were prepared by inoculating YEMA broth medium from 24 hours old slant. Liquid cultures were incubated on a rotary shaker at $28^{\circ} \mathrm{C}$ for 48 hours. 
The soil was sterilized by $\mathrm{NaOCl} 2.5 \%$, and then washed several times with distilled water, air dried and distributed into $10 \mathrm{Kg}$ portions in plastic pots $10 \mathrm{Cm}$ diameter. Five seeds from each faba bean variety were sown in each pot.

Table (3): Physical and chemical properties of the soil used

\section{Genotypic characterization:}

Isolation of genomic DNA from rhizobial isolates:

Genomic DNA of rhizobial cells was isolated and purified using GeneJetTM Genomic DNA purification Kit (Fermentas ${ }^{\circledR}$, Thermo Scientific, EU). The procedures were carried out according to manufacture instructions: Rhizobial cells up to $1 \times 10^{9}$ were harvested and resuspended in $180 \mu$ l digestion solution. Twenty microliters of proteinase $\mathrm{K}$ was added and incubated at $56^{\circ} \mathrm{C}$ for $30 \mathrm{~min}$. Twenty microliters of ribonuclease (RNase) solution was added and incubated for $10 \mathrm{~min}$ at room temperature. Two hundreds microliters of lysis solution were added and mixed well by vortex for $20 \mathrm{sec}$. Four hundreds microliters of $50 \%$ ethanol were added, transferred to GeneJet ${ }^{\text {TM }}$ Genomic DNA purification column and was centrifuged for $1 \mathrm{~min}$ at $8000 \mathrm{xg}$. The column was washed twice by washing buffer I and washing buffer II, separately. Finally purified genomic DNA was eluted by elution buffer. Genomic DNA was electrophorised on $0.7 \%$ agarose gel, stained by ethidium bromide and visualized by gel documentation system (BIO-RAD ${ }^{\circledR}$, USA).

\begin{tabular}{|c|c|}
\hline Soil property & value \\
\hline $\begin{array}{l}\text { Mechanical analysis: } \\
\text { Coarse sand \% } \\
\text { Fine sand \% } \\
\text { Silt \% } \\
\text { Clay \% } \\
\text { Soil texture }\end{array}$ & $\begin{array}{l}4.75 \\
30-20 \\
25-70 \\
39.35 \\
\text { Clay loam }\end{array}$ \\
\hline $\begin{array}{l}\text { Physical properties: } \\
\text { Saturation Percentage (S.P. \%) } \\
\text { PH } \\
\text { E.C. }\left(\mathrm{dsm}^{-1} \mathbf{2 5}^{\circ} \mathrm{C}\right) \\
\text { Organic Matter (O.M. \%) } \\
\text { Total nitrogen (T.N. \%) } \\
\text { CaCO3 \% }\end{array}$ & \begin{tabular}{l|}
55.0 \\
7.6 \\
5.06 \\
0.38 \\
0.10 \\
2.20
\end{tabular} \\
\hline $\begin{array}{l}\text { Chemical properties: } \\
\text { Soluble cations (meq/L) } \\
\mathrm{Ca}^{++} \\
\mathrm{Mg}^{++} \\
\mathrm{Na}^{+} \\
\mathrm{K}^{+} \\
\text {Soluble anions (meq/L) } \\
\mathrm{CO3}^{--} \\
\mathrm{HCO3}^{-} \\
\mathrm{Cl}^{-} \\
\mathrm{SO}^{--}\end{array}$ & $\begin{array}{l}13.0 \\
12.0 \\
28.5 \\
0.28 \\
\\
\overline{1.4} \\
25.0 \\
27.38\end{array}$ \\
\hline
\end{tabular}

Amplification and sequencing of 16S Rrna gene:

In the present study, universal primers 27 F (5'-AGAGTTTGATCCTGGCTCAG-3') and 1492R (5'GGTTACCTTGTTACGACTT-3') (Lane, 1991) were used to amplify nearly full-length $16 \mathrm{~S}$ rRNA gene (1500 bp). PCR was performed using the standard reaction mixture $(50 \mu \mathrm{l})$ containing: $1 \mathrm{X}$ PCR buffer, $1.5 \mathrm{mM} \mathrm{MgCl} 2,5 \%$ dimethyl sulfoxide, $200 \mathrm{mM}$ of each dNTPs, 15 pmol of each primer, $1 \mathrm{U}$ of Taq polymerase enzyme and $50 \mathrm{ng}$ of DNA template. PCR reaction conditions were; an initial denaturation at $95^{\circ} \mathrm{C}$ for $3 \mathrm{~min}, 35$ cycles of denaturation at $94^{\circ} \mathrm{C}$ for $30 \mathrm{sec}$, annealing at $60^{\circ} \mathrm{C}$ for $30 \mathrm{sec}$., and extension at $72{ }^{\circ} \mathrm{C}$ for $1.5 \mathrm{~min}$ final extension at $72{ }^{\circ} \mathrm{C}$ for $7 \mathrm{~min}$ (Shuhaimi, 2003). Reactions were performed in PCR Thermo cycler machine (BIO-RAD ${ }^{\circledR}$, USA). PCR products were separated on $1 \%$ agarose gel. The amplified fragments of $16 \mathrm{~S}$ rDNA were purified using QIAGEN ${ }^{\circledR}$ PCR purification kit $\left(Q_{\text {IAGEN }}^{\circledR}\right.$, Germany) according to the instructions of the manufacture. DNA sequencing was carried out using 3100 Genetic Analyzer (Applied Biosystem, USA) based on the enzymatic chain terminator technique developed by Sanger et al., (1977). 
DNA reagents:

50X TAE buffer (the working concentration is $1 \mathrm{X}$ )

Tris base............242g

EDTA $(0.5 \mathrm{M}) \ldots \ldots \ldots . .37 .2 \mathrm{~g}$

Glacial acetic acid.......57.1 ml

Distilled water...... Up to $1000 \mathrm{ml}$.

Gel loading dye (for agarose)

Sucrose........40.0g

Bromophenol blue........ $0.25 \mathrm{~g}$

Xylene cyanol FF.......... $0.25 \mathrm{~g}$

Distilled water...... Up to $100 \mathrm{ml}$.

Ethidium bromide

$5 \mathrm{mg} / \mathrm{ml}$ in distilled $\mathrm{H} 2 \mathrm{O}(50.000 \mathrm{X})$

Primers for 16S rRNA (Lane1991)

-27F (5'-AGAGTTTGATCCTGGCTCAG-3')

-1492R (5'-GGTTACCTTGTTACGACTT-3')

\section{Results and discussion:-}

Isolation and characterization of rhizobia:-

Sixteen isolates of rhizobia were recovered from the root nodules of faba bean (Vicia faba L.) grown in different soil locations and properties as well as various climatic conditions in Egypt. Pure colonies of rhizobial isolates were microscopically examined to determine cell shape, size, Gram stain reaction and motility in liquid culture. Colony morphology of rhizobial isolates was studied to determine the transparency and viscosity by using binuclear microscope. Data in table (4) clearly showed that all rhizobial isolates tested were fast growing on YEMA medium, non capsulated, short rod, Gram negative and motile. Cell size ranged from 1 to $4 \mathrm{~mm}$ among all tested isolates. The most isolates colonies are opaque (10 isolates) while (6) isolates were translucent. Six of the tested isolates recorded in low viscosity and four isolates recorded in very high viscosity while the other isolates gave high viscosity. In this respect the obtained data are in harmony with data reported by Bergey's Manual of systematic Bacteriology (2005), Garrity, (2005), who reported that the characteristics of rhizobia were short rod (0.5-1.0 x 1.2-3.0 $\mu \mathrm{m})$, non spore forming, Gram negative reaction and motile by 1-6 peritrichous flagella. Colonies are usually white or beige, crawlers, convex, semi-translucent or opaque raised and mucilaginous usually 2-4 $\mathrm{mm}$ in diameter. 
Table 4:-Some morphological characteristics of rhizobial isolates

\begin{tabular}{|c|c|c|c|c|c|c|c|c|}
\hline & \multirow{2}{*}{$\begin{array}{l}\text { Source of } \\
\text { Isolate } \\
\text { Origin }\end{array}$} & \multirow{2}{*}{$\begin{array}{l}\text { Code of } \\
\text { isolate }\end{array}$} & \multicolumn{3}{|c|}{ Cell morphology } & \multicolumn{3}{|c|}{ Morphology of colony } \\
\hline & & & Shape & $\begin{array}{l}\text { Gram } \\
\text { reaction }\end{array}$ & Motility & $\begin{array}{l}\text { Size } \\
(\mathbf{m m})\end{array}$ & Transparency & Viscosity \\
\hline 1 & $\begin{array}{l}\text { El- } \\
\text { Menoufia }\end{array}$ & K-ISORH1 & $\begin{array}{l}\text { Short } \\
\text { rods }\end{array}$ & negative & + & $1-2$ & Trans L. & Low \\
\hline 2 & Qena & $\begin{array}{l}\text { K-ISORH } \\
2\end{array}$ & $\begin{array}{l}\text { Short } \\
\text { rods }\end{array}$ & negative & + & $1-2$ & Trans L. & Low \\
\hline 3 & Qena & K-ISORH3 & $\begin{array}{l}\text { Short } \\
\text { rods }\end{array}$ & negative & + & $1-2$ & Opaque & High \\
\hline 4 & Matana & K-ISORH4 & $\begin{array}{l}\text { Short } \\
\text { rods }\end{array}$ & negative & + & $2-3$ & Opaque & High \\
\hline 5 & Matana & K-ISORH5 & $\begin{array}{l}\text { Short } \\
\text { rods }\end{array}$ & negative & + & $1-2$ & Opaque & High \\
\hline 6 & $\begin{array}{l}\text { El- } \\
\text { Menoufia }\end{array}$ & K-ISORH6 & $\begin{array}{l}\text { Short } \\
\text { rods }\end{array}$ & negative & + & $1-2$ & Trans L. & Low \\
\hline 7 & Giza & K-ISORH7 & $\begin{array}{l}\text { Short } \\
\text { rods }\end{array}$ & negative & + & $2-3$ & Opaque & Very High \\
\hline 8 & Kalubia & K-ISORH8 & $\begin{array}{l}\text { Short } \\
\text { rods }\end{array}$ & negative & + & $1-2$ & Opaque & High \\
\hline 9 & El-Minia & K-ISORH9 & $\begin{array}{l}\text { Short } \\
\text { rods }\end{array}$ & negative & + & $2-4$ & Trans L. & Low \\
\hline 10 & El-Behaira & $\begin{array}{l}\text { K- } \\
\text { ISORH10 }\end{array}$ & $\begin{array}{l}\text { Short } \\
\text { rods }\end{array}$ & negative & + & $1-2$ & Opaque & Very High \\
\hline 11 & $\begin{array}{l}\text { El- } \\
\text { Dakahlia }\end{array}$ & $\begin{array}{l}\text { K- } \\
\text { ISORH11 }\end{array}$ & $\begin{array}{l}\text { Short } \\
\text { rods }\end{array}$ & negative & + & $2-3$ & Opaque & High \\
\hline 12 & Kalubia & $\begin{array}{l}\text { K- } \\
\text { ISORH12 }\end{array}$ & $\begin{array}{l}\text { Short } \\
\text { rods }\end{array}$ & negative & + & $1-2$ & Opaque & Very High \\
\hline 13 & Kalubia & $\begin{array}{l}\text { K- } \\
\text { ISORH13 }\end{array}$ & $\begin{array}{l}\text { Short } \\
\text { rods }\end{array}$ & negative & + & $2-3$ & Opaque & High \\
\hline 14 & Giza & $\begin{array}{l}\text { K- } \\
\text { ISORH14 }\end{array}$ & $\begin{array}{l}\text { Short } \\
\text { rods }\end{array}$ & negative & + & $1-2$ & Trans L. & Low \\
\hline 15 & Baniswaif & $\begin{array}{l}\text { K- } \\
\text { ISORH15 }\end{array}$ & $\begin{array}{l}\text { Short } \\
\text { rods }\end{array}$ & negative & + & $2-3$ & Opaque & Very High \\
\hline 16 & Assiut & $\begin{array}{l}\text { K- } \\
\text { ISORH16 }\end{array}$ & $\begin{array}{l}\text { Short } \\
\text { rods }\end{array}$ & negative & + & $1-2$ & Trans L. & Low \\
\hline
\end{tabular}

\begin{tabular}{|l|l|l|}
\hline & Isolates & $\mathrm{NaCl} \%$ \\
\hline
\end{tabular}




\begin{tabular}{|c|c|c|c|c|c|c|c|c|c|c|c|c|c|c|c|}
\hline & & 0.0 & 0.5 & 1.0 & 1.5 & 2.0 & 2.5 & 3.0 & 3.5 & 4.0 & $\begin{array}{l}4 . \\
5\end{array}$ & $\begin{array}{l}5 . \\
0\end{array}$ & $\begin{array}{l}5 . \\
5\end{array}$ & $\begin{array}{l}6 . \\
0\end{array}$ & $\begin{array}{l}6 . \\
5\end{array}$ \\
\hline 1 & $\begin{array}{l}\text { K- } \\
\text { ISORH1 }\end{array}$ & $\begin{array}{l}++ \\
+\end{array}$ & $\begin{array}{l}++ \\
+\end{array}$ & $\begin{array}{l}++ \\
+\end{array}$ & $\begin{array}{l}++ \\
+\end{array}$ & $\begin{array}{l}++ \\
+\end{array}$ & $\begin{array}{l}++ \\
+\end{array}$ & $\begin{array}{l}++ \\
+\end{array}$ & ++ & ++ & + & + & - & - & - \\
\hline 2 & $\begin{array}{l}\text { K-ISORH } \\
2\end{array}$ & $\begin{array}{l}++ \\
+\end{array}$ & $\begin{array}{l}++ \\
+\end{array}$ & $\begin{array}{l}++ \\
+\end{array}$ & $\begin{array}{l}++ \\
+\end{array}$ & $\begin{array}{l}++ \\
+\end{array}$ & $\begin{array}{l}++ \\
+\end{array}$ & $\begin{array}{l}++ \\
+\end{array}$ & ++ & ++ & + & - & - & - & - \\
\hline 3 & $\begin{array}{l}\text { K- } \\
\text { ISORH3 }\end{array}$ & $\begin{array}{l}++ \\
+\end{array}$ & $\begin{array}{l}++ \\
+\end{array}$ & $\begin{array}{l}++ \\
+\end{array}$ & $\begin{array}{l}++ \\
+\end{array}$ & $\begin{array}{l}++ \\
+\end{array}$ & $\begin{array}{l}++ \\
+\end{array}$ & $\begin{array}{l}++ \\
+\end{array}$ & ++ & + & - & - & - & - & - \\
\hline 4 & $\begin{array}{l}\text { K- } \\
\text { ISORH4 }\end{array}$ & $\begin{array}{l}++ \\
+\end{array}$ & $\begin{array}{l}++ \\
+\end{array}$ & $\begin{array}{l}++ \\
+\end{array}$ & $\begin{array}{l}++ \\
+\end{array}$ & $\begin{array}{l}++ \\
+\end{array}$ & $\begin{array}{l}++ \\
+\end{array}$ & ++ & + & - & - & - & - & - & - \\
\hline 5 & $\begin{array}{l}\text { K- } \\
\text { ISORH5 }\end{array}$ & $\begin{array}{l}++ \\
+\end{array}$ & $\begin{array}{l}++ \\
+\end{array}$ & $\begin{array}{l}++ \\
+\end{array}$ & $\begin{array}{l}++ \\
+\end{array}$ & $\begin{array}{l}++ \\
+\end{array}$ & $\begin{array}{l}++ \\
+\end{array}$ & ++ & ++ & + & - & - & - & - & - \\
\hline 6 & $\begin{array}{l}\text { K- } \\
\text { ISORH6 }\end{array}$ & $\begin{array}{l}++ \\
+\end{array}$ & $\begin{array}{l}++ \\
+\end{array}$ & $\begin{array}{l}++ \\
+\end{array}$ & $\begin{array}{l}++ \\
+\end{array}$ & $\begin{array}{l}++ \\
+\end{array}$ & $\begin{array}{l}++ \\
+\end{array}$ & $\begin{array}{l}++ \\
+\end{array}$ & ++ & ++ & + & + & - & - & - \\
\hline 7 & $\begin{array}{l}\text { K- } \\
\text { ISORH7 }\end{array}$ & $\begin{array}{l}++ \\
+\end{array}$ & $\begin{array}{l}++ \\
+\end{array}$ & $\begin{array}{l}++ \\
+\end{array}$ & $\begin{array}{l}++ \\
+\end{array}$ & $\begin{array}{l}++ \\
+\end{array}$ & $\begin{array}{l}++ \\
+\end{array}$ & $\begin{array}{l}++ \\
+\end{array}$ & $\begin{array}{l}++ \\
+\end{array}$ & ++ & ++ & + & + & + & - \\
\hline 8 & $\begin{array}{l}\text { K- } \\
\text { ISORH8 }\end{array}$ & $\begin{array}{l}++ \\
+\end{array}$ & $\begin{array}{l}++ \\
+\end{array}$ & $\begin{array}{l}++ \\
+\end{array}$ & $\begin{array}{l}++ \\
+\end{array}$ & $\begin{array}{l}++ \\
+\end{array}$ & $\begin{array}{l}++ \\
+\end{array}$ & $\begin{array}{l}++ \\
+\end{array}$ & ++ & ++ & + & + & + & - & - \\
\hline 9 & $\begin{array}{l}\text { K- } \\
\text { ISORH9 }\end{array}$ & $\begin{array}{l}++ \\
+\end{array}$ & $\begin{array}{l}++ \\
+\end{array}$ & $\begin{array}{l}++ \\
+\end{array}$ & $\begin{array}{l}++ \\
+\end{array}$ & $\begin{array}{l}++ \\
+\end{array}$ & $\begin{array}{l}++ \\
+\end{array}$ & $\begin{array}{l}++ \\
+\end{array}$ & ++ & ++ & + & + & - & - & - \\
\hline 10 & $\begin{array}{l}\text { K- } \\
\text { ISORH10 }\end{array}$ & $\begin{array}{l}++ \\
+\end{array}$ & $\begin{array}{l}++ \\
+\end{array}$ & $\begin{array}{l}++ \\
+\end{array}$ & $\begin{array}{l}++ \\
+\end{array}$ & $\begin{array}{l}++ \\
+\end{array}$ & $\begin{array}{l}++ \\
+\end{array}$ & ++ & ++ & ++ & + & + & - & - & - \\
\hline 11 & $\begin{array}{l}\text { K- } \\
\text { ISORH11 }\end{array}$ & $\begin{array}{l}++ \\
+\end{array}$ & $\begin{array}{l}++ \\
+\end{array}$ & $\begin{array}{l}++ \\
+\end{array}$ & $\begin{array}{l}++ \\
+\end{array}$ & $\begin{array}{l}++ \\
+\end{array}$ & $\begin{array}{l}++ \\
+\end{array}$ & $\begin{array}{l}++ \\
+\end{array}$ & ++ & ++ & + & + & + & - & - \\
\hline 12 & $\begin{array}{l}\text { K- } \\
\text { ISORH12 }\end{array}$ & $\begin{array}{l}++ \\
+\end{array}$ & $\begin{array}{l}++ \\
+\end{array}$ & $\begin{array}{l}++ \\
+\end{array}$ & $\begin{array}{l}++ \\
+\end{array}$ & $\begin{array}{l}++ \\
+\end{array}$ & $\begin{array}{l}++ \\
+\end{array}$ & $\begin{array}{l}++ \\
+\end{array}$ & ++ & ++ & + & + & - & - & - \\
\hline 13 & $\begin{array}{l}\text { K- } \\
\text { ISORH13 }\end{array}$ & $\begin{array}{l}++ \\
+\end{array}$ & $\begin{array}{l}++ \\
+\end{array}$ & $\begin{array}{l}++ \\
+\end{array}$ & $\begin{array}{l}++ \\
+\end{array}$ & ++ & ++ & ++ & ++ & + & - & - & - & - & - \\
\hline 14 & $\begin{array}{l}\text { K- } \\
\text { ISORH14 }\end{array}$ & $\begin{array}{l}++ \\
+\end{array}$ & $\begin{array}{l}++ \\
+\end{array}$ & $\begin{array}{l}++ \\
+\end{array}$ & $\begin{array}{l}++ \\
+\end{array}$ & $\begin{array}{l}++ \\
+\end{array}$ & $\begin{array}{l}++ \\
+\end{array}$ & $\begin{array}{l}++ \\
+\end{array}$ & ++ & + & - & - & - & - & - \\
\hline 15 & $\begin{array}{l}\text { K- } \\
\text { ISORH15 }\end{array}$ & $\begin{array}{l}++ \\
+\end{array}$ & $\begin{array}{l}++ \\
+\end{array}$ & $\begin{array}{l}++ \\
+\end{array}$ & $\begin{array}{l}++ \\
+\end{array}$ & $\begin{array}{l}++ \\
+\end{array}$ & $\begin{array}{l}++ \\
+\end{array}$ & $\begin{array}{l}++ \\
+\end{array}$ & $\begin{array}{l}++ \\
+\end{array}$ & $\begin{array}{l}++ \\
+\end{array}$ & ++ & + & + & + & - \\
\hline 16 & $\begin{array}{l}\text { K- } \\
\text { ISORH16 }\end{array}$ & $\begin{array}{l}++ \\
+\end{array}$ & $\begin{array}{l}++ \\
+\end{array}$ & $\begin{array}{l}++ \\
+\end{array}$ & ++ & ++ & ++ & ++ & ++ & ++ & + & + & - & - & - \\
\hline 17 & ARC-202 & $\begin{array}{l}++ \\
+\end{array}$ & $\begin{array}{l}++ \\
+\end{array}$ & $\begin{array}{l}++ \\
+\end{array}$ & $\begin{array}{l}++ \\
+\end{array}$ & $\begin{array}{l}++ \\
+\end{array}$ & $\begin{array}{l}++ \\
+\end{array}$ & $\begin{array}{l}++ \\
+\end{array}$ & ++ & + & + & - & - & - & - \\
\hline 18 & $\begin{array}{l}\text { ICARDA } \\
-441\end{array}$ & $\begin{array}{l}++ \\
+\end{array}$ & $\begin{array}{l}++ \\
+\end{array}$ & $\begin{array}{l}++ \\
+\end{array}$ & $\begin{array}{l}++ \\
+\end{array}$ & $\begin{array}{l}++ \\
+\end{array}$ & $\begin{array}{l}++ \\
+\end{array}$ & ++ & + & - & - & - & - & - & - \\
\hline 19 & $\begin{array}{l}\text { ICARDA } \\
-481 \\
\end{array}$ & $\begin{array}{l}++ \\
+ \\
\end{array}$ & $\begin{array}{l}++ \\
+ \\
\end{array}$ & $\begin{array}{l}++ \\
+ \\
\end{array}$ & $\begin{array}{l}++ \\
+ \\
\end{array}$ & $\begin{array}{l}++ \\
+ \\
\end{array}$ & $\begin{array}{l}++ \\
+ \\
\end{array}$ & $\begin{array}{l}++ \\
+ \\
\end{array}$ & ++ & + & - & - & - & - & - \\
\hline
\end{tabular}

Table 4:-Some morphological characteristics of rhizobial isolates

Trans L. $=$ Translucent 
Table 5:-The effect of addition of different $\mathrm{NaCl}$ concentrations on the growth of rhizobial isolates grown on YAM medium

$$
\begin{aligned}
& \text { ( }) \text { No growth } \\
& (++) \text { good growth }\left(10^{7}-10^{8}\right. \text { cfu per ml) }
\end{aligned}
$$

(+) Poor growth ( $\geq 10^{7}$ cfu per $\left.\mathrm{ml}\right)$ $(+++)$ very good growth $\left(\leq 10^{9}\right.$ cfu per $\left.\mathrm{ml}\right)$

\section{The effect of different $\mathrm{NaCl}$ concentrations on growth of rhizobial isolates:-}

Data in table (5) summarized the effect of various $\mathrm{NaCl}$ concentrations (from 0.0 to $6.5 \%$ ) on the sixteen rhizobial isolates. The all tested grew very well up to $2.5 \% \mathrm{NaCl}$ concentration, while their growth was varied on $\mathrm{NaCl}$ concentrations from $3.0 \%$ to $4.0 \%$. No growth was found at $\mathrm{NaCl} 6.5 \%$ concentration among the all tested isolates. Only K-RHISO7 and K- RHISO15 had a positive growth at 4.5 till $6.0 \% \mathrm{NaCl}$ concentration. These results are in agreement with (Keneni, et al., 2010; Belal, et al., 2013 and Mona, H., 2017), who found that rhizobial isolates were able to grow up to $5 \% \mathrm{NaCl}$ concentration and not completely inhibited. They added the rhizobial growth was not affected by low and medium level as salinity ( $\mathrm{NaCl} \%$ concentration).

Data in table (5) clearly showed that both of the isolates K-ISORH7 and K-ISORH15 succeeded to grow in elevated salt concentrations where they showed observed growth till $\mathrm{NaCl}$ concentration of $6.0 \%$. So they are considered the most potent and tolerant isolated.

\section{Isolates and nodulation status as well as plant growth: Isolates and nodulation status:}

Nodules number (no plant ${ }^{-1}$ ), nodules dry weight (mg plant ${ }^{-1}$ ) and nitrogenase activity (ml mole plant ${ }^{-1}$ hour $^{-1}$ ) of 16 rhizobial isolates as well as 3 reference rhizobial strains results are presents in table (6). The obtained data illustrated that the all tested rhizobial isolates had nodules formation on the roots of the two faba bean varieties used and scored nodules number values ranged from 10 to 188 nodules per plant and recorded significant differences between the various rhizobial isolates namely K-ISORH13 recorded the lowest nodules number per plant (10 nodules) in contrast K-ISORH11, 7 and 15 having the highest nodules number and these values were 139 , 172 and 188 nodules per plant respectively at faba bean (V1) and the corresponding values at (V2) were 98, 122 and 125 in the same order, while the rhizobial reference strains recorded nodules number ranged from 62 to 125 no plant ${ }^{-1}$ and rhizobial strains ICARDA-441 having highest nodules number (125 no plant $\left.{ }^{-1}\right)$. Irrespective of faba bean varieties used, the highest nodules number values were recorded at K-ISORH15 (157) followed by K-ISORH7 (147).

Data present in table (6) indicated that the nodules dry weight of the obtained rhizobial isolates and / or rhizobial references strains had the same trend which obtained at nodules number. The highest nodule dry weight were found at K-ISORH7, 11 and 15 and these values were 597, 421 and $607 \mathrm{mg}$ per plant respectively for faba bean variety G843 (V1) and the corresponding values for faba bean variety Sakha-1 (V2) were 395, 297 and 404 in the same order. The lowest values were found at K-ISORH13 (63 mg/plant) at variety G-843 and K-ISORH9 (82 mg / plant) at variety Sakha-1. For reference rhizobial strains tested, the ICARDA-441 gave the highest nodule dry weight and recorded 396 and $330 \mathrm{mg}$ / plant for G-843 and Sakha-1 faba bean varieties respectively, as compared to other tested reference strains. Nitrogenase activity, the all tested rhizobial isolates and strains had a positive effect and scored nitrogenase activity ( $\mathrm{N}$-ase) among isolates these values ranged from 1.03 to 81.1 , while at reference strains were 1.1 to $10.36 \mathrm{ml} \mathrm{mole} \mathrm{plant}^{-1}$ hour $^{-1}$. The highest nitrogenase values were found at K-ISORH15 (81.1) followed KISORH7 (45.6) with G-843 faba bean variety. In general with regardless the varieties used the highest nitrogenase $\left(\mathrm{N}\right.$-ase) values scored were 50.1 and $39.8 \mathrm{ml} \mathrm{mole} \mathrm{plane}{ }^{-1}$ hour $^{-1}$ at K-ISORH15 and K-ISORH7 respectively, As compared to other tested rhizobial isolates and strain. From the above mentioned data, faba bean variety G- 843 was superior as compared to variety Sakha-1 in the all tested parameters where recorded 69 and 230 for nodule number and nodule dry weight respectively, and the corresponding values at variety Sakha-1 were 59 and 185 in the same order. In this respect, the obtained results are in harmony with (Tsyganov, et al., 2002; Sprent, 2007; Peter, et al., 2013 and Mona, 2017) who reported that the legume-rhizobia symbiosis is well known as a highly specific mutually beneficial plant-microbe interaction, which means that certain legume species can only successfully interact with certain strains of rhizobia and they added that the inoculated legume plants with different rhizobial isolates and strains led to scoring the higher values of nodule number and dry weight per plant as well as nitrogenase activity for the nodules formed. Also, Digvijay, et al., (2011), reported that the native rhizobial strains gave a positive response between plant-bacteria relationship as well as eco-soil system and they added to get new rhizobial strains. 


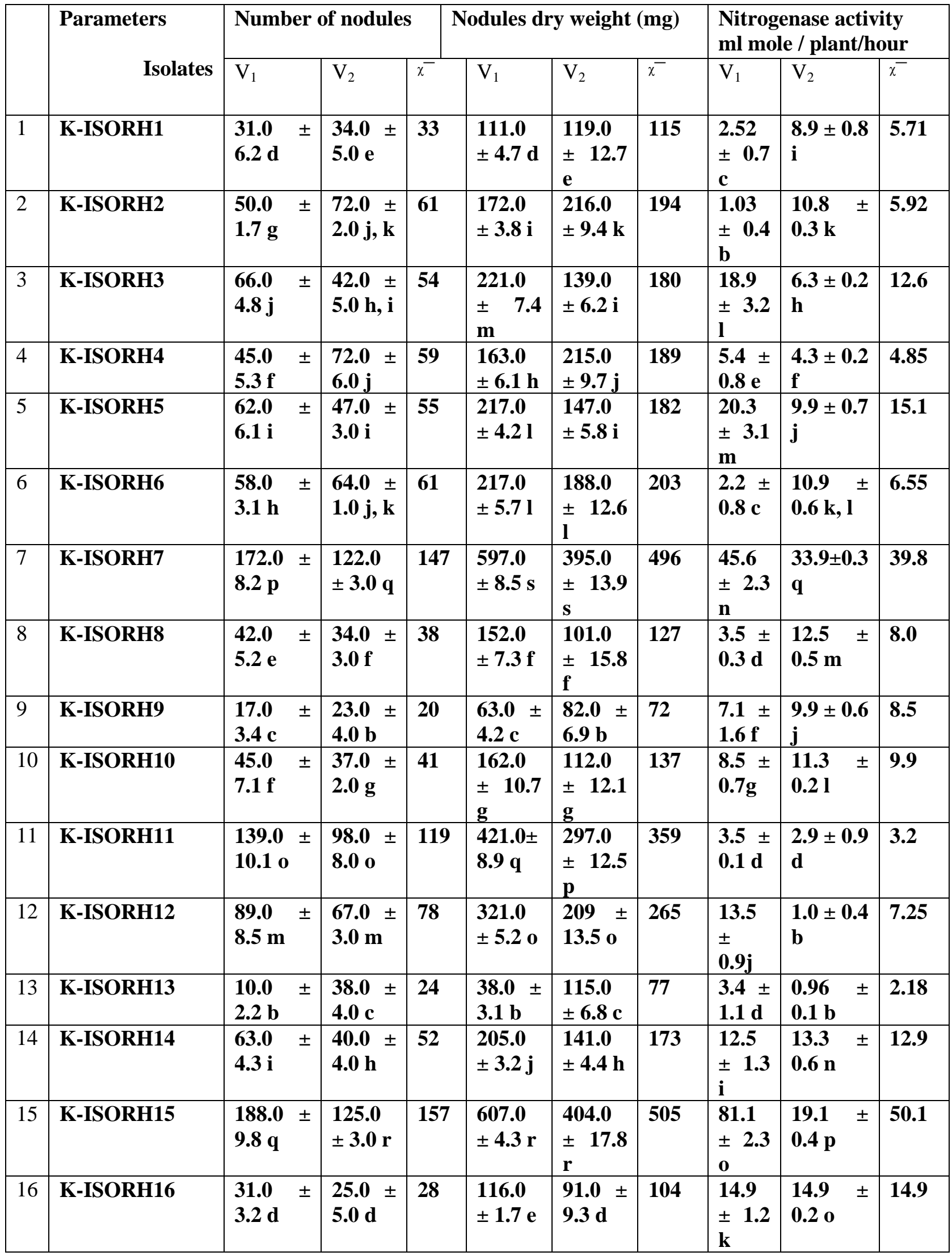




\begin{tabular}{|c|c|c|c|c|c|c|c|c|c|c|}
\hline$\chi^{-}$ & & 69.0 & 59.0 & 64.0 & 236.4 & 185.6 & 211.1 & 15.25 & 10.68 & 12.97 \\
\hline 17 & ARC-202 & $\begin{array}{l}80.0 \pm \\
4.0 k\end{array}$ & $\begin{array}{l}22.0 \pm \\
5.0 \mathrm{k}\end{array}$ & 15 & $\begin{array}{l}10.0 \pm \\
2.0 \mathrm{k}\end{array}$ & $\begin{array}{ll}100.0 \\
\pm \quad 4.0 \\
m\end{array}$ & 55 & $\begin{array}{l}9.93 \\
\pm 2.1 \\
\text { h }\end{array}$ & $\begin{array}{l}5.3 \pm 0.5 \\
\mathrm{~g}\end{array}$ & 7.62 \\
\hline 18 & ICARDA-441 & $\begin{array}{l}125.0 \pm \\
3.6 \mathrm{n}\end{array}$ & $\begin{array}{l}\text { 53.0 } \pm \\
2.0 \mathrm{n}\end{array}$ & 39 & $\begin{array}{l}9.0 \pm \\
1.1 p\end{array}$ & $\begin{array}{l}190.0 \\
\pm 4.3 \mathrm{q}\end{array}$ & 99.5 & $\begin{array}{ll}10.36 \\
\pm 1.4 \\
\text { h }\end{array}$ & $\begin{array}{l}3.7 \pm 0.4 \\
\text { e }\end{array}$ & 7.03 \\
\hline 19 & ICARDA-481 & $\begin{array}{l}83.0 \pm \\
4.21\end{array}$ & $\begin{array}{l}\text { 43.0 } \pm \\
3.01\end{array}$ & 25.5 & $\begin{array}{l}110.0 \\
\pm 4.0 \mathrm{n}\end{array}$ & $\begin{array}{l}120.0 \\
\pm \quad 30.0 \\
\text { n }\end{array}$ & 115 & $\begin{array}{l}1.1 \pm \\
0.8 \mathrm{~b}\end{array}$ & $\begin{array}{l}1.98 \quad \pm \\
0.98 \text { c }\end{array}$ & 1.54 \\
\hline
\end{tabular}

Table 6:-Nodules number, dry weight and nitrogenase activity of 16 various rhizobial isolates and 3 reference rhizobial strains at 45 DAP

\section{Isolates and plant growth:-}

Data of shoot and root dry weight of faba bean plants $\left(\mathrm{gm} \mathrm{plant}^{-1}\right)$ as affected by application of 16 rhizobial isolates as well as 3 reference rhizobial strains are shown in table (7). The obtained results illustrated that the all tested rhizobial isolates and/or strains gave significant differences at shoots and roots dry weight. Faba bean plants recorded shoot dry weight ranged from 1.7 to $3.3 \mathrm{gm}_{\text {plant }}{ }^{-1}$ and root dry weight from 0.5 to $1.0 \mathrm{gm} \mathrm{plant}^{-1}$ among the all tested isolates and strains with the two faba bean varieties \{G843 (V1) and Sakha1 (V2)\} tested. The highest shoot dry weights were recorded at rhizobial isolates namely K-ISORH5, K-ISORH7 and K-ISORH15 and these values were 3.0,3.1 and 3.3 gm plant $^{-1}$ respectively for faba bean variety G843 and the corresponding values for the same isolates for faba bean variety Sakhal were 2.1, 2.5 and $2.7 \mathrm{gm} \mathrm{plant}^{-1}$ in the same order.

The results of faba bean root dry weight at table (7) had the same trend which obtained at shoot dry weight and rhizobial isolates namely K-ISORH5, K-ISORH7 and K-ISORH15 scored the highest root dry weight (gm plant ${ }^{-1}$ ) and these values were $0.9,0.9$ and $1.0 \mathrm{gm}^{-1 a n t^{-1}}$ respectively at $\mathrm{V} 1$ and the corresponding values at V2 were 0.7, 1.0 and $1.0 \mathrm{gm} \mathrm{plant}^{-1}$ in the same order.

From the above mentioned data, faba bean variety G843 was superior as compared to variety Sakha1 in both shoot and root dry weight of faba bean plants.

Generally, irrespective to the two faba bean varieties tested, the two rhizobial K-ISORH7 and K-ISORH15 gave the highest shoot and root plants dry weights as compared to other tested rhizobial isolates and strains and recorded 2.8 and $3.0 \mathrm{gm} \mathrm{plant}^{-1}$ for shoot dry weight respectively, and for roots dry weight were 1.0 and $1.0 \mathrm{gm} \mathrm{plant}^{-1}$ in the same order.

In this respect, the obtained results are in agreement with (EL-Zanaty, et al., 2005; Workalemahu, 2009; Digvijay, et al., (2011), Zahran, et al., 2012 and Mona, et al., 2016). Who reported that the isolated native rhizobial strains are of good traits also tolerant to high salt levels, have the ability to form root nodules in high number as well as to induce host plant to give good vegetative growth ( shoot and root dry weight).

From the above mentioned data it is clearly shown that the both two rhizobial isolates K-ISORH7 and K-ISORH15 as well as faba bean variety G843 were superior as compared to other tested rhizobial isolates and faba bean varieties at all nodulation and vegetative growth tested parameters. 
Table(7):- The effect of rhizobial inoculation with 16 isolates as well as 3 reference strains on shoots and roots dry weight of faba bean plants

\begin{tabular}{|c|c|c|c|c|c|c|c|}
\hline & Parameters & $\begin{array}{l}\text { Shoots dry } \\
\text { (gm) }\end{array}$ & eight & & $\begin{array}{l}\text { Roots dry } \\
\text { (gm) }\end{array}$ & & \\
\hline & & $\mathrm{V}_{1}$ & $\mathrm{~V}_{2}$ & $x^{-}$ & $\mathrm{V}_{1}$ & $\mathrm{~V}_{2}$ & $x^{-}$ \\
\hline 1 & K-ISORH1 & $2.8 \pm 0.1 \mathrm{k}$ & $2.5 \pm 0.4 \mathrm{~h}$ & 2.65 & $0.6 \pm 0.2 \mathrm{~b}$ & $0.7 \pm 0.6 \mathrm{~d}$ & 0.65 \\
\hline 2 & K-ISORH2 & $\begin{array}{l}2.1 \pm 0.3 \mathrm{f}, \\
\mathrm{g}\end{array}$ & $2.0 \pm 0.2 \mathrm{~d}, \mathrm{e}$ & 2.05 & $0.7 \pm 0.1 \mathrm{c}$ & $0.7 \pm 0.4 \mathrm{~d}$ & 0.7 \\
\hline 3 & K-ISORH3 & $2.6 \pm 0.4 \mathrm{i}$ & $1.9 \pm 0.3 \mathrm{c}, \mathrm{d}$ & 2.25 & $0.9 \pm 0.2 \mathrm{e}$ & $0.6 \pm 0.3 \mathrm{c}$ & 0.75 \\
\hline 4 & K-ISORH4 & $2.9 \pm 0.2 \mathrm{l}$ & $2.1 \pm 0.2 \mathrm{e}, \mathrm{f}$ & 2.5 & $0.8 \pm 0.2 \mathrm{~d}$ & $0.6 \pm 0.4 \mathrm{~b}$ & 0.7 \\
\hline 5 & K-ISORH5 & $3.0 \pm 0.1 \mathrm{~m}$ & $2.1 \pm 0.3 \mathrm{e}, \mathrm{f}$ & 2.55 & $0.9 \pm 0.1 \mathrm{e}$ & $0.7 \pm 0.2 \mathrm{~d}$ & 0.8 \\
\hline 6 & K-ISORH6 & $1.7 \pm 0.3 \mathrm{c}$ & $1.7 \pm 0.4 \mathrm{~b}$ & 1.7 & $0.6 \pm 0.2 \mathrm{~b}$ & $0.7 \pm 0.3 \mathrm{~d}$ & 0.65 \\
\hline 7 & K-ISORH7 & $3.1 \pm 0.1 \mathrm{n}$ & $2.5 \pm 0.1 \mathrm{~h}$ & 2.8 & $0.9 \pm 0.1 \mathrm{f}$ & $1.0 \pm 0.2 \mathrm{~g}$ & 1.0 \\
\hline 8 & K-ISORH8 & $1.5 \pm 0.2 \mathrm{~b}$ & $1.9 \pm 0.3 \mathrm{c}, \mathrm{d}$ & 1.7 & $0.6 \pm 0.3 \mathrm{~b}$ & $0.7 \pm 0.2 \mathrm{~d}$ & 0.65 \\
\hline 9 & K-ISORH9 & $2.1 \pm 0.5 f$ & $1.8 \pm 0.5 \mathrm{c}, \mathrm{d}$ & 1.95 & $0.7 \pm 0.1 \mathrm{c}$ & $0.5 \pm 0.4 \mathrm{a}$ & 0.6 \\
\hline 10 & K-ISORH10 & $2.5 \pm 0.1 \mathrm{~h}$ & $2.0 \pm 0.4 \mathrm{~d}, \mathrm{e}$ & 2.25 & $0.8 \pm 0.2 \mathrm{~d}$ & $0.5 \pm 0.2 \mathrm{a}$ & 0.65 \\
\hline 11 & K-ISORH11 & $2.0 \pm 0.3 \mathrm{e}$ & $2.3 \pm 0.2 \mathrm{~g}$ & 2.15 & $0.8 \pm 0.09 d$ & $0.7 \pm 0.1 \mathrm{~d}$ & 0.75 \\
\hline 12 & K-ISORH12 & $2.5 \pm 0.2 \mathrm{~h}$ & $2.7 \pm 0.4 \mathrm{i}$ & 2.6 & $0.8 \pm 0.3 \mathrm{~d}$ & $0.7 \pm 0.2 \mathrm{~d}$ & 0.75 \\
\hline 13 & K-ISORH13 & $2.7 \pm 0.1 \mathrm{j}$ & $2.6 \pm 0.7 \mathrm{~h}, \mathrm{i}$ & 2.65 & $0.8 \pm 0.2 \mathrm{~d}$ & $0.6 \pm 0.1 \mathrm{~b}$ & 0.7 \\
\hline 14 & K-ISORH14 & $2.0 \pm 0.4 \mathrm{e}$ & $2.0 \pm 0.2 \mathrm{~d}, \mathrm{e}$ & 2.0 & $0.7 \pm 0.2 \mathrm{c}$ & $0.6 \pm 0.2 \mathrm{~b}$ & 0.65 \\
\hline 15 & K-ISORH15 & $3.3 \pm 0.10$ & $2.7 \pm 0.2 \mathrm{i}$ & 3.0 & $1.0 \pm 0.1 \mathrm{e}$ & $0.9 \pm 0.2 \mathrm{f}$ & 1.0 \\
\hline 16 & K-ISORH16 & $2.2 \pm 0.3 \mathrm{~g}$ & $2.5 \pm 0.4 \mathrm{~h}$ & 2.35 & $0.7 \pm 0.2 \mathrm{c}$ & $0.8 \pm 0.2 \mathrm{e}$ & 0.75 \\
\hline$x$ & & 2.44 & 2.2 & 2.32 & 0.77 & 0.69 & 0.73 \\
\hline 17 & ARC-202 & $2.5 \pm 0.3 h$ & $2.7 \pm 0.2 \mathrm{i}$ & 2.6 & $0.8 \pm 0.2 \mathrm{~d}$ & $0.7 \pm 0.6 d$ & 1.4 \\
\hline 18 & ICARDA-441 & $2.6 \pm 0.2 \mathrm{i}$ & $2.2 \pm 0.1 \mathrm{f}, \mathrm{g}$ & 2.4 & $0.7 \pm 0.3 \mathrm{c}$ & $0.7 \pm 0.3 \mathrm{~d}$ & 1.20 \\
\hline 19 & ICARDA-481 & $1.8 \pm 0.2 \mathrm{~d}$ & $1.9 \pm 0.3 \mathrm{c}, \mathrm{d}$ & 1.85 & $0.6 \pm 0.4 \mathrm{~b}$ & $0.6 \pm 0.3 \mathrm{~b}$ & 1.6 \\
\hline
\end{tabular}

Genotypic characterization of the efficient rhizobial isolates

DNA isolation

Genomic DNA of rhizobial isolates was isolated using Wizard ${ }^{\circledR}$ Genomic DNA purification Kit, promega ${ }^{\circledR}$ Corporation, USA. Fig (1) 


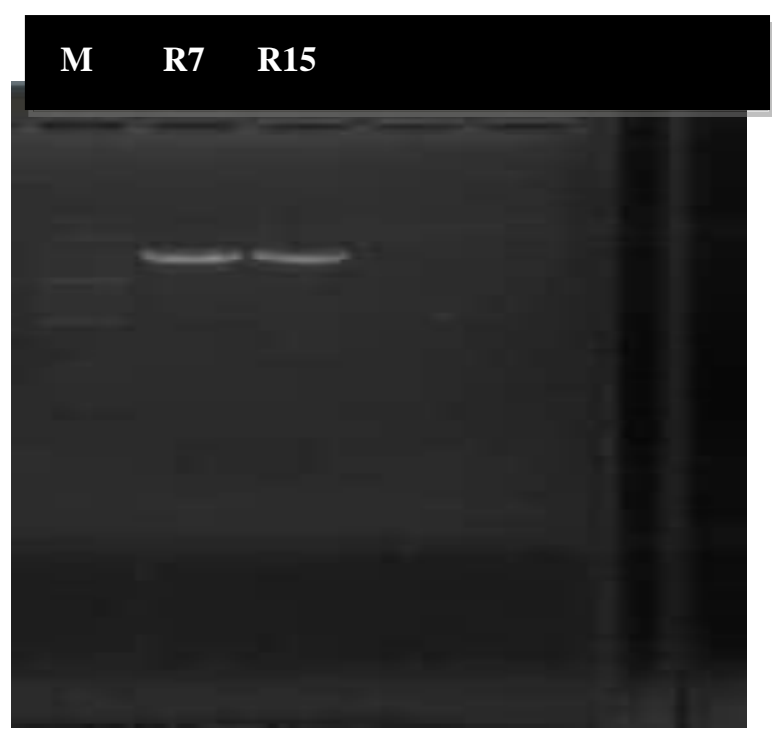

Figure 1:-purified genomic DNA of rhizobial isolates. Lane M: 1kb plus DNA marker, Fermentas ${ }^{\circledR}$, lane R7: KISORH7 and lane R15: K-ISORH15

\section{PCR amplification of 16S rRNA gene}

PCR amplification of $16 \mathrm{~S}$ rRNA gene $(1500$ bp) was done using published primers $27 \mathrm{~F}$ (5'AGAGTTTGATCCTGGCTCAG-3') and 1492R (5'-GGTTACCTTGTTACGACTT-3'), Lane, (1991). The PCR products were further cleaned up and purified using QIAquick Gel extraction kit, Qiagen ${ }^{\circledR}$ according to the kit manual instruction. Fig (2).

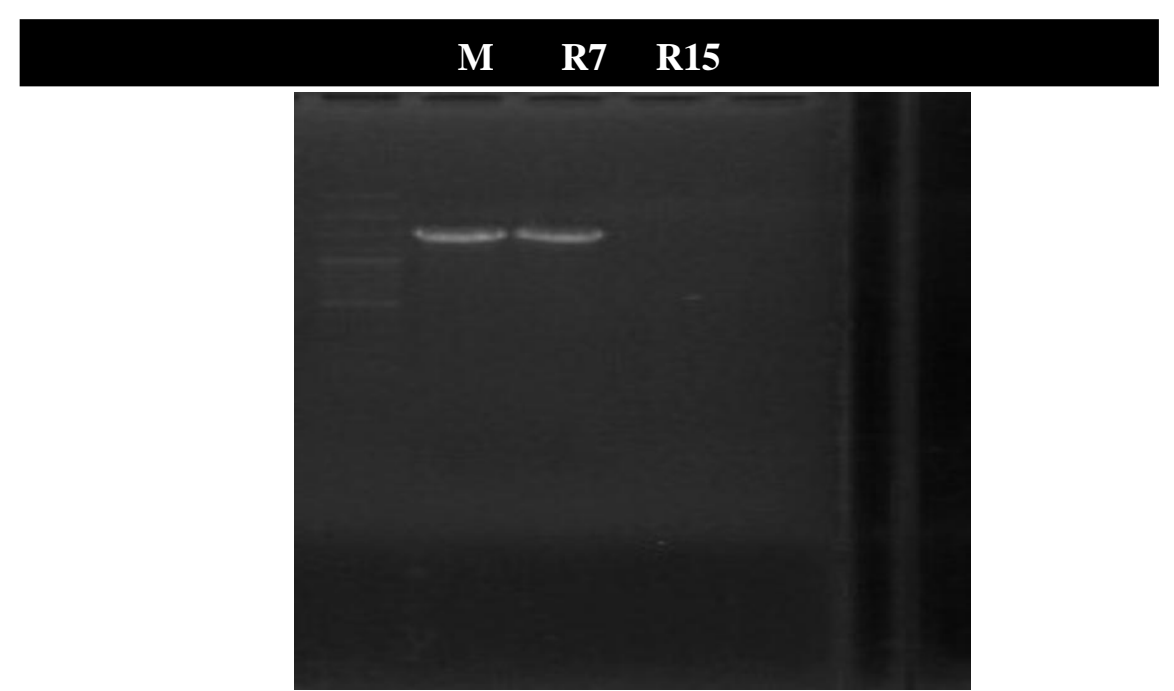

Figure 2:-16S Rrna PCR products (1500 bp) of rhizobial isolates. Lane M: 100 b plus DNA marker, Fermentas ${ }^{\circledR}$, lane R7: K-ISORH7 and lane R15: K-ISORH15

Table 8:-Bacterial isolates used in this study and their phyllogenetic homology

\begin{tabular}{|c|l|l|l|l|}
\hline \multirow{2}{*}{$\begin{array}{c}\text { Rhizobial } \\
\text { isolates }\end{array}$} & $\begin{array}{l}\text { NCBI } \\
\text { Accession }\end{array}$ & $\begin{array}{l}\text { Identity based on 16S rRNA gene sequence } \\
(\text { bp) }\end{array}$ & Closest species (accession number) & $\begin{array}{l}\text { Identity } \\
(\%)\end{array}$ \\
\hline $\begin{array}{c}\text { K- } \\
\text { ISORH7 }\end{array}$ & LC431741 & 1284 & Rhizobium sp. HR7(KM276562) & 100 \\
$\begin{array}{c}\text { K- } \\
\text { ISORH15 }\end{array}$ & LC431742 & $\mathbf{1 3 1 8}$ & $\begin{array}{l}\text { Rhizobium sp. strain SEMIA 439 } \\
\text { (MH236581) }\end{array}$ & 100 \\
\hline
\end{tabular}


According to $16 \mathrm{~S}$ rRNA sequence, isolates were identified as Rhizobium sp. By $100 \%$ similarity with HR7(KM276562) and strain SEMIA 439 (MH236581) for K-ISORH7 and K-ISORH15 respectively with accession numbers LC431741 and LC431742 in the same order as shown in table (8). The sequences of the tested strains are found in details at the electronic web site (http://www.ncbi.nlm.nih.gov) .

\section{Conclusion:-}

It can be concluded that the native rhizobia succeeded to form nodules on roots of faba bean plants. From this study, the bacterial rhizobial isolates were superior to give number, dry weight and nitrogenase activity for obtained nodules as compared to reference strains used. Moreover, the most potent isolates K-ISORH7 and K-ISORH15, identified genetically and found similarity as Rhizobium sp. By 100\% similarity with HR7(KM276562) and strain SEMIA 439 (MH236581) for K-ISORH7 and K-ISORH15 respectively.

\section{References:-}

1. Belal, E. B.; Hassan, M. M. and El-Ramady, H. R. (2013): Phylogenetic and characterization of sat-tolerant rhizobial strain nodulating fababean plants. Afri. J. Biotechnol. 27: 4324-4337.

2. Bond, D. A.; Lawes, D.;A.; Hawtin, G.C.; Saxena, M. C. and Stephens, J. S. (1985): Faba Bean (Vicia faba L.). p. 199-265. In: R.J. Summerfield and E.H. Roberts (eds.), Grain Legume Crops. William Collins Sons Co. Ltd. 8 Grafton Street, London, WIX 3LA, UK.

3. Digvijay, P.; Peter, S. and Subrata, K.D. (2011): Rhizobium pusense sp., isolated from the rhizosphere of chickpea (Cicerarietinum L.).

4. Inter. J.of Syst. and Evol.Microbiology. 61: 2632-2639.

5. El-Zanaty, A.M.; Hewedy, O.A.; Nagaty, H.H. and Abdelbary, M. I.(2005): Molecular and biochemical characterization of some Egyptian genotypes. Bioeng. and Biomed. Sci. 5: 2155-2238.

6. Garrity, G. M., Leventhal J., and Lilburn T. G. (2005): posting date. Taxonomic Outline of the Procaryotes, Bergey's Manual of Systematic Bacteriology, Second Edition,. Springer. [Online.]

7. Keneni, A.; Assefa, F. and Prabu, P.C. (2010): Characterization of acid and salt-tolerant Emir. J. Food Agric. 25: $102-108$.

8. Lane, D.J.(1991): 16S/23S rRNA sequencing. In Stackebrandt, E; Goodfellow, M (Eds.) Nucleic Acid Techniques in Bact. Syst.. New York, Wiley, 115-175.

9. Mona, H. A. (2017): Improving faba bean productivity using biofertlization under stress conditios. Thesis. phD in agric. science Agric. botany dep., faculty of agriculture. Benha univ.44-80.

10. Mona, H. A.; Zaghloul, R. A.; Abou Aly, H. A.; Abdel-Rahman, H. M. and Abotaleb, H. H. (2016): Isolation and Identification of rhizobial strains from faba bean nodules. Annals of Agric. Sci., 54: 591-600.

11. Peter, M. V.; Duncan, N. L. M.; Sasha C. R. and Cory C. C. (2013): Biological nitrogen fixation: rates, patterns and ecological controls in terrestrial ecosystems. Downloaded from rstb.royalsocietypublishing.org

12. Pooja, S. and Rajesh, K. (2015): Soil salinity: A serious environmental issue and plant growth promoting bacteria as one of the tools for its alleviation. Saudi J. Biol. Sci. 22: 123-131.

13. Sanger, F.; Nicklen, S. and Coulson, A. R. (1977): "DNA sequencing with chain terminating inhibitors". Proceedings of the National Academy of Sciences of the United States of America, 74: 5463-5467.

14. Shahbaz M. and Ashraf M. (2013): Improving salinity tolerance in cereals. Crit. Rev. Plant Sci. 32: $237-249$.

15. Shuhaimi, M. (2003): Species classification and molecular studies of bile salt hydrolase gene in Bifidobacterium spp. Doctor of philosophy. University. Putra, Malaysia, 1-174.

16. Somasegaran P. and Hoben H. J. (1994): Handbook for rhizobia: methods in legume-rhizobium technology. Springer-Verlag, New York USA.

17. Sprent J.I. and James E.K. (2007): Legume evolution: where do nodules and mycorrhizas fit in. Plant Physiol. 144: 575-581.

18. Tsyganov, V. E.; Voroshilova, V. A.; Priefer, U. B.; Borisov, A.Y.and Tikhonovich, I.A. (2002): Genetic dissection of the initiation of the infection process and nodule tissue development in the Rhizobium-pea (Pisum sativum L.) symbiosis. Ann. Bot. 89: 357-366.

19. Vincent, J.M. (1970): A manual for the practical study of the root nodulebacteria, IBP Handbook No. 15, Oxford: Blackwell Scientific Publishers.

20. Workalemahu, A. (2009): The effect of indigenous root-nodulating bacteria on nodulation and growth of faba bean (Vicia faba) in the low-input agricultural systems od Tigary Highlands, Northern Ethiopia. Mol. Rev. 63: 968-989. 
21. Yamaguchi, T. and Blumwald, E. (2005): Developing salt-tolerant crop plants: challenges and opportunities. Trends Plant Sci. $10: 615-620$.

22. Zahran, H.H.; Abdel-Fattah, M.; Yassen, M. M.; Mahmoud, A. M. and Bedmar, E. J. (2012): Diversity and environmental stress responses of rhizobial bacteria from Egyptian grain legumes. Aust. J.of Basic and App. Sci.6: 571-583.

23. https://www.hunker.com/12328257/importance-of-legumes

24. http://www.ncbi.nlm.nih.gov

25. http://passel.unl.edu/pages/informationmodule.php?idinformationmodule=956783940\&topicorder $=10 \& \operatorname{maxto}=$ 10. 